\title{
REMOTE SENSING CLASSIFICATION USING MULTI-SENSOR SUPER-RESOLUTION ALGORITHM
}

\author{
Alexander Belov and Anna Denisova \\ Samara National Research University \\ 34, Moskovskoye shosse, Samara, 443086, Russia
}

\begin{abstract}
Super-resolution image fusion aims to produce an image with finer spectral and spatial details than the input images. However, the super-resolution fusion is mainly applied to enhance a visual representation of the images and its potential benefits to the final thematic classification is an open question. In this paper, we present an experimental investigation of the remote sensing image classification performance in the case of the multi-sensor super-resolution image fusion. The research aims to compare classification performance obtained for the fused image and the low resolution input ones using different standard-of-the-art classifiers and feature extraction methods. Input data are supposed to be multispectral data obtained in visible and near infrared spectral ranges by the different remote sensing systems. To perform a multi-sensor super-resolution image fusion, we used a gradient-descent optimization approach with a B-TV regularization successfully adapted for remote sensing images with different spatial and spectral sampling characteristics by the authors of the paper. As for features, we applied brightness in spectral channels, attribute profiles and local feature attribute profiles. The classification was performed using support vector machines and random forest classifiers that have been proved to be very effective for remote sensing data classification. The experimental research included the multi-sensor input data simulation for four remote sensing systems, the super-resolution image fusion of all simulated images and the thematic classification of the fused image and the images obtained as an average input for each of the simulated imaging systems. The spatial resolution of the fused image was in 2, 3,4 and 5 times better than the spatial resolution of the modeled input images. The average bandwidth of the fused image was $29 \mathrm{~nm}$ whereas for the input low resolution images it was in the range from 37 to $83 \mathrm{~nm}$. Experimental results have shown that random forest classification is better to use with fusion, whereas support vector machines demonstrated better results without fusion. The feature extraction test showed that extended attribute profiles enhance the random forest classification accuracy of the fused image. Thus, the classification results have shown that super-resolution image fusion leads to the classification accuracy increase in the case of random forest classifier and there is no need to apply fusion in the case of support vector machines.
\end{abstract}

\section{KEYWORDS}

Image Classification, Data Fusion, Super-Resolution, SVM, RF

\section{INTRODUCTION}

Remote sensing data fusion aims to enhance image quality in terms of spectral, temporal or spatial resolution. Among all super-resolution fusion algorithms, the most prominent algorithms seem to be spectral-spatial super-resolution algorithms which enhance both spectral and spatial resolution of the images simultaneously. Despite the evident visual enhancement of the images caused by spectral-spatial super-resolution algorithms, the potential effect on the image classification remains an open question. Therefore, our study aims to assess classification quality after spectral-spatial super-resolution.

In our study, we applied our own spectral-spatial super-resolution algorithm. The algorithm provides super-resolution image fusion in multi-frame case when input low resolution images (LR images) are supposed to be captured by different remote sensing systems with the different spectral and spatial sampling parameters (Belov and Denisova, 2018). The algorithm finds the estimate of the high resolution image (HR image) using the predefined spatial and spectral sampling parameters. We estimated the classification quality of the fused image using two well-known classifiers: Support Vector Machines with Radial Basis Functions (SVM-RBF) and Random Forest (RF). As shown in papers (Tuia et al.,2014; Belgiu and Drăguţ, 2016; Li et al.,2016; Khatami et al., 2016; García et al., 2019; Liao et al., 2015), these classifiers are the most 
frequently used for remote sensing data especially in the case of small training sets. As for features, we regarded brightness in spectral channels and two additional comprehensive feature extraction methods: Extended Attribute Profiles (EAP) and Local Feature Attribute Profiles (LFAP). The latter two feature extraction methods were effectively applied in remote sensing data analysis in papers (Pham et al., 2017; Pham, Aptoula and Lefèvre, 2018; Pham et al., 2018; Hong et al., 2020).

The goal of our experimental research was to answer the question whether the super-resolution image fusion is able to enhance image classification. We made our experimental research using simulated remote sensing data of four different remote sensing systems. The data for all of the four systems were used in the fusion process with the predefined parameters of the output spectral and spatial resolution. As a result, we assessed the classification quality in two cases for the fused image and for average input images of each system.

The rest of the paper is organized in the following way. Section 2 provides a description of our super-resolution image fusion algorithm. Section 3 describes the data simulation process. Section 4 contains a fusion parameters description and classification description. Section 5 concerns the experimental results.

\section{SPECTRAL-SPATIAL SUPER-RESOLUTION IMAGE FUSION ALGORITHM}

The described fusion algorithm was proposed in (Belov and Denisova, 2018) and it develops the ideas of the spatial super-resolution of the color images introduced by Fausiu et al. in (Farsiu et al., 2004, Farsiu et al. 2003). In contrast to the algorithms proposed in (Farsiu et al., 2004, Farsiu et al. 2003), our algorithm provides spectral resolution enhancement as well as the spatial one. In this paper, the spectral resolution is defined by the number of spectral channels and their average bandwidth.

The algorithm exploits the following observation model to describe the relation between input LR images and the reference HR image:

$$
\begin{aligned}
& Y_{k \xi l}=D_{k} F_{k \xi} H_{k}\left(\sum_{\lambda=1}^{L} w_{k l}(\lambda) X_{\lambda}\right)+V_{k \xi l}, \\
& k=1, \ldots, K, l=1, \ldots, L_{k}, \xi=1, \ldots, \xi_{k},
\end{aligned}
$$

where $X_{\lambda}$ is the $\lambda$-th spectral channel of the reference HR image, $Y_{k \xi \xi l}$ is the $l$-th spectral channel of the $\xi$-th LR image obtained by the k-th imaging system, $w_{k \xi}(\lambda)$ are the coefficients of the spectral transformation from L-channel reference spectral representation into $L_{k}$-th observed spectral representation of the k-th imaging system. Operator $H_{k}$ defines an optical blur for the $k$-th system. Operator $F_{k \xi}$ is a frame geometry and frame motion transformation caused by the georeferencing errors. $F_{k \xi}$ is defined relatively to the reference frame position corresponding to the HR image $X_{\lambda}$. The operator $D_{k}$ is the uniform sampling with averaging over the square are $T_{k} \times T_{k}$ where $T_{k}$ is a ground sampling distance (GSD). The operators $H_{k}, F_{k \xi}$ and $D_{k}$ are supposed to be linear and finite. $V_{k \xi \xi l}$ is the Gaussian white noise in the $l$-th spectral channel of the image $Y_{k \xi}$. It is assumed that the GSD of the reference HR image is less than the GSDs of the input LR images $T<T_{k}, k=1, \ldots, K$, and the reference number of spectral channels is larger than the maximum channel number of the input LR images $L \geq \max _{k=1, \ldots, K}\left(L_{k}\right)$.

The fusion algorithm provides a gradient descent optimization of the following task:

$$
\begin{aligned}
& \hat{X}=\underset{X}{\arg \min }\left\{\sum_{k=1}^{K} \sum_{l=1}^{L_{k}}\left\|\frac{1}{\xi_{k}} \sum_{\xi=1}^{\xi_{k}} F_{k \xi}^{-1} I_{k} \sum_{\lambda=1}^{L} w_{k l}(\lambda) D_{k} H_{k} F_{k \xi} X_{\lambda}-Z_{k l}\right\|_{1}+\beta \sum_{i=-p}^{p} \sum_{j=-p}^{p} \alpha^{|i|+|j|}\left\|X_{L}-S_{1}^{i} S_{2}^{j} X_{L}\right\|_{1}\right\} \\
& Z_{k l}=\frac{1}{\xi_{k}} \sum_{\xi=1}^{\xi k} F_{k \xi}^{-1} I_{k}\left(Y_{k \xi l}\right), k=1, \ldots, K, l=1, \ldots, L_{k}
\end{aligned}
$$


where $Z_{k l}$ is the $l$-th spectral channel of the average image $Z_{k}$ for the $k$-th imaging system, $I_{k}$ is the interpolation operator with the sampling step $T / T_{k}, F_{k \xi}^{-1}$ is the inverse operator of the frame geometry and frame motion transformation of the $\xi$-th observed image for the $k$-th imaging system. Both operators $I_{k}$ and $F_{k \xi}^{-1}$ are assumed to be linear. Parameters $p, \alpha, \beta$ determine the weights of the B-TV regularization term.

The optimization task (2) is solved using the gradient descent method and results in the estimate $\hat{X}$ of the reference image with the sampling parameters $T, L$.

\section{DATA SIMULATION}

Experimental research was conducted using simulated data of four different imaging systems. We used 10 fragments of hyperspectral images as source data for simulation. All image fragments were derived from the images of the Hyperspectral scenes dataset (Graña et al., 2020) which have ground truth information. The source hyperspectral image fragments are described in Table 1.

Table 1. Hyperspectral image fragments used for simulation

\begin{tabular}{llll}
\hline Name & Size & Number of classes & Spectral range, $\mathbf{n m}$ \\
\hline Botswana-1 & $240 \times 240$ & 6 & $447,17-1057,68$ \\
Botswana-2 & $240 \times 240$ & 5 & $447,17-1057,68$ \\
Pavia-1 & $420 \times 420$ & 8 & $430-808,75$ \\
Pavia-2 & $420 \times 420$ & 6 & $430-808,75$ \\
Pavia-3 & $420 \times 420$ & 5 & $430-808,75$ \\
PaviaU-1 & $240 \times 240$ & 6 & $430-812,5$ \\
PaviaU-2 & $240 \times 240$ & 8 & $430-812,5$ \\
PaviaU-3 & $240 \times 240$ & 8 & $430-812,5$ \\
Salinas-1 & $180 \times 180$ & 8 & $365,93-1262,96$ \\
Salinas-2 & $180 \times 180$ & 8 & $365,93-1262,96$ \\
\hline
\end{tabular}

The hyperspectral image fragments were used for the reference multispectral HR image simulation $X$ and for the observed LR image $Y_{k \xi}, k=1, \ldots, 4, \xi=1, \ldots, \xi_{k}$ simulation according to the observation model (1).

Spectral channels of the simulated images were determined using Gaussian spectral response function (SRF) $W_{l}(u)$ with the full width at half maximum (FWHM) and central wavelength $u_{l}^{0}$ parameters:

$$
W_{l}(u)=\delta_{l}^{-1}(2 \pi)^{-1 / 2} \exp \left\{-0.5\left(u-u_{l}^{0}\right)^{2} / \delta_{l}^{2}\right\},
$$

where $F W H M=2 \sqrt{2 \ln (2)} \delta_{l}$ and $l$ is the number of spectral channel.

Without loss of generality, we assumed that the GSD of the HR image is the same as the GSD of the hyperspectral image fragment and it is equal to $T=1$. The number of spectral channels of the reference HR image was $\mathrm{L}=15$ for all images except Pavia and PaviaU images for which it was $\mathrm{L}=11$. The parameters of the spectral channels of the reference HR image are shown in Table 2.

Table 2. Parameters of the reference image spectral channels

\begin{tabular}{llllllllllllllll}
\hline № & 1 & 2 & 3 & 4 & 5 & 6 & 7 & 8 & 9 & 10 & 11 & 12 & 13 & 14 & 15 \\
\hline$u_{l}$ & 463 & 492 & 521 & 550 & 580 & 609 & 638 & 665 & 694 & 724 & 753 & 782 & 812 & 841 & 870 \\
FWHM & 29 & 29 & 29 & 29 & 29 & 29 & 29 & 29 & 29 & 29 & 29 & 29 & 30 & 30 & 30 \\
\hline
\end{tabular}

The GSDs $T_{k}$ of four imaging systems were 2, 3, 4 and 5 correspondingly. The spectral channel configuration for the LR images was selected as spectral channels of four different real remote sensing systems: Geoton (Resurs-P), Spot-7, Kanopus-B and Sentinel-2A. Table 3 shows the parameters of the spectral channels for simulated imaging systems. 
Table 3. Parameters of spectral channels for simulated imaging systems

\begin{tabular}{lllllllll}
\hline \multirow{2}{*}{$\underline{ }$} & \multicolumn{3}{c}{$\mathrm{k}=1$} & $\mathrm{k}=2$ & & $\mathrm{k}=3$ & \multicolumn{2}{c}{$\mathrm{k}=4$} \\
\cline { 2 - 9 } & $u_{0}$ & FWHM & $u_{0}$ & FWHM & $u_{0}$ & FWHM & $u_{0}$ & FWHM \\
\hline 1 & 485 & 70 & 490 & 70 & 490 & 30 & 490 & 65 \\
2 & 560 & 80 & 560 & 60 & 555 & 45 & 560 & 35 \\
3 & 645 & 70 & 660 & 70 & 660 & 30 & 665 & 30 \\
4 & 685 & 30 & 825 & 130 & 795 & 45 & 705 & 15 \\
5 & 715 & 30 & - & - & - & - & 740 & 15 \\
6 & 750 & 100 & - & - & - & - & 783 & 20 \\
7 & - & - & - & - & - & - & 842 & 115 \\
8 & - & - & - & - & - & - & 865 & 20 \\
\hline
\end{tabular}

Since the hyperspectral image fragments for images Pavia and PaviaU had smaller spectral range, the simulation for these images was made without spectral channels typed with an italic font in tables 2 and 3 .

The reference HR image was generated as a result of spectral sampling of the hyperspectral image fragment.

LR images were generated according to the model (1). The coefficients of the spectral transformation for $\mathrm{k}$-th imaging system were determined according to Gaussian SRFs (4) in the following way:

$$
w_{k l}(\lambda)=\int_{\zeta_{1}(\lambda)}^{\zeta_{2}(\lambda)} W_{k l}(u) d u / \int_{-\infty}^{+\infty} W_{k l}(u) d u,
$$

where $\lambda$ is the number of reference spectral channel, $l$ is the number of channel for the k-th system, $\zeta_{1}(\lambda)=u_{k l}^{0}-F W H M, \zeta_{2}(\lambda)=u_{k l}^{0}+F W H M$

The frame geometry and frame motion operator $F_{k \xi}$ was determined as a transient shift on $\chi_{\xi 1}$ and $\chi_{\xi 2}$ pixels in the horizontal and vertical directions:

$X_{k \xi}^{F}\left(n_{1}, n_{2}, l\right)=F_{k \xi}\left(X_{k}^{W}\left(n_{1}, n_{2}, l\right)\right)=X_{k}^{W}\left(n_{1}+\chi_{\xi 1}, n_{2}+\chi_{\xi 2}, l\right)$.

Operator $H_{k}$ was defined as:

$X_{k \xi}^{H}\left(n_{1}, n_{2}, l\right)=H_{k}\left(X_{k \xi}^{F}\left(n_{1}, n_{2}, l\right)\right)=\sum_{\tau_{1}=-\Delta k}^{\Delta k} \sum_{\tau_{2}=-\Delta k}^{\Delta k} h\left(\tau_{1}, \tau_{2}\right) X_{k \xi}^{F}\left(n_{1}-\tau_{1}, n_{2}-\tau_{2}, l\right)$,

where $h\left(\tau_{1}, \tau_{2}\right)=A \exp \left(-0.5 \sigma_{k}^{-2}\left(\tau_{1}^{2}+\tau_{2}^{2}\right)\right)$ is an impulse response of the Gaussian blur with radius $\sigma_{k}$.

The spatial sampling $D_{k}$ was defined as two sequential operators of averaging $D_{1}$ and decimation $D_{2}$ :

$$
\begin{aligned}
& X_{k \xi}^{D 1}\left(n_{1}, n_{2}, l\right)=D_{k 1}\left(X_{k \xi}^{H}\left(n_{1}, n_{2}, l\right)\right)=\frac{1}{T_{k}^{2}} \sum_{\tau_{1}=-\lceil T k / 2\rceil}^{\lceil T k / 2\rceil} \sum_{\tau_{2}=-\lceil T k / 2\rceil}^{\lceil T k / 2\rceil} X_{k \xi}^{H}\left(n_{1}-\tau_{1}, n_{2}-\tau_{2}, l\right), \\
& X_{k \xi}^{D 2}\left(m_{1}, m_{2}, l\right)=D_{k 2}\left(X_{k \xi}^{D 1}\left(n_{1}, n_{2}, l\right)\right)=\left.X_{k \xi}^{D 1}\left(n_{1}, n_{2}, l\right)\right|_{\substack{n_{1}=m_{1} T_{k} \\
n_{2}=m_{2} T_{k}}} .
\end{aligned}
$$

Table 4 contains parameters of spatial distortions for each of the simulated imaging systems.

Table 4. Spatial distortions parameters for the simulated imaging systems

\begin{tabular}{lllll}
\hline Parameter & $k=1$ & $k=2$ & $k=3$ & $k=4$ \\
\hline$\chi_{\xi 1}, \chi_{\xi 3}$ & {$[-2,2]$} & {$[-3,3]$} & {$[-4,4]$} & {$[-5,5]$} \\
$\sigma_{k}$ & 5 & 7 & 9 & 11 \\
$T_{k}$ & 2 & 3 & 4 & 5 \\
\hline
\end{tabular}


The examples of the reference multispectral HR images are shown in Figure 1.

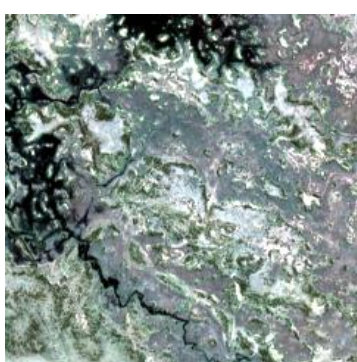

a)

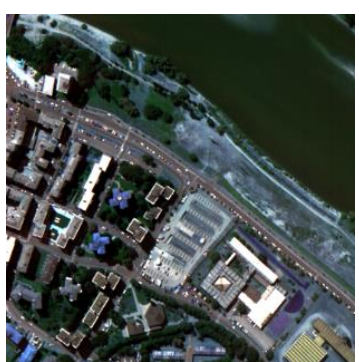

b)

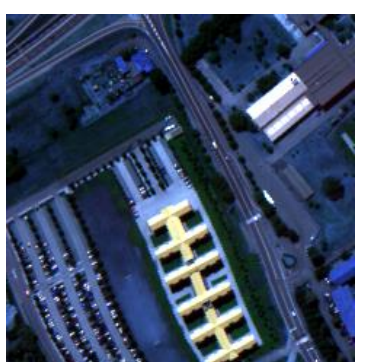

c)

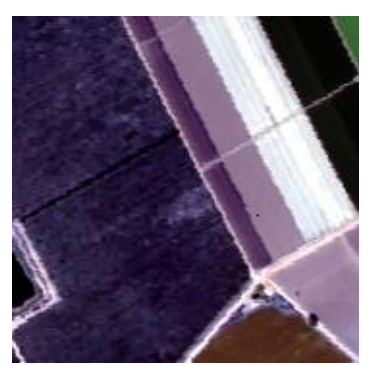

d)

Figure 1. The examples of the reference multispectral HR images: a) Botswana-1, b) Pavia-1, c) PaviaU-1, d) Salinas-1

\section{FUSION AND CLASSIFICATION PARAMETERS}

The fusion was carried out using the algorithm (Belov and Denisova, 2018) with the following regularization parameters: $\alpha_{k}=0.1, p=2$ and $\beta=0.5$. The number of gradient descent method iterations was equal to 280. The step of the gradient descent process was 20 .

We used the images of four systems for fusion. The number of the observed images for each imaging system was determined according to the rule of the optimal multi-frame image super-resolution (Farsiu et al., 2003) and it was equal to 4, 9, 16, 25 for the system 1, system 2, system 3 and system 4 correspondingly, the examples of fusion result and average input images for each system are shown in Figure 2.

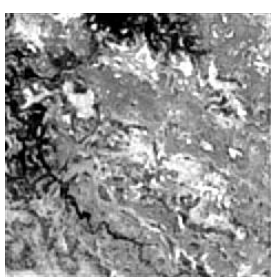

a)

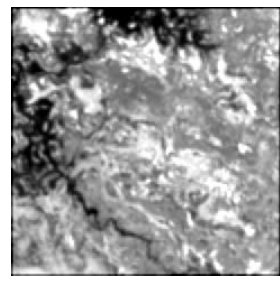

b)

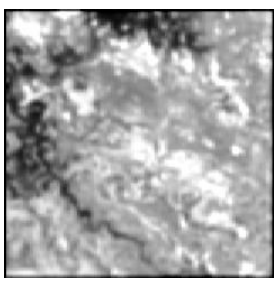

c)

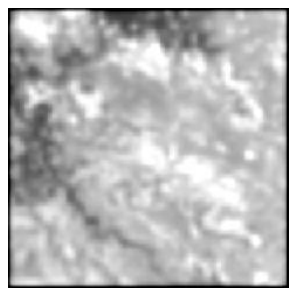

d)

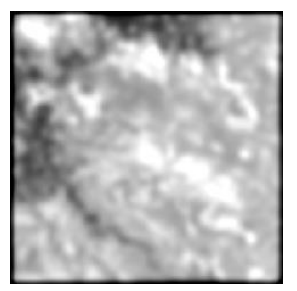

e)

Figure 2. The examples of: a) fusion result, b) average image for system $1, \mathrm{c}$ ) average image for system 2 , d) average image for system 3 , e) average image for system 4

To make the fused image and average images for each system comparable, we made the following kind of preprocessing. The input LR images for each of the $\mathrm{K}$ systems were transformed into average images $Z_{k}$ using formula (3). For this purpose, we applied nearest neighbor interpolation and inverse frame motion operator with zero padding. Due to the zero padding, the images $Z_{k}$ contained the lower values of pixels along the borders. Therefore, to make fused and average images comparable we reduced the size of all images in such a way that there were no incorrect border pixels in average images for each of the imaging systems.

The reference classification masks were divided into training and control sets with the ratio 7:3. The maximum number of pixels per class was equal to 15 . The examples of the reference classification masks are shown in Figure 3.

Classification and feature extraction were performed using Matlab packages developed in studies (Marpu et al., 2013; Li et al., 2015). The SVM and RF algorithms were used with default parameters.

Classification accuracy was measured using the probability of true classification $P$ that is defined as the ratio of correctly classified pixels to the total number of pixels in the test set. Further, we use average $P$ value for 5 runs of the classification. 


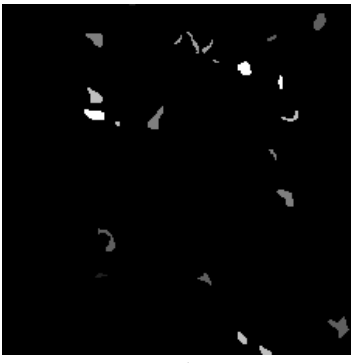

a)

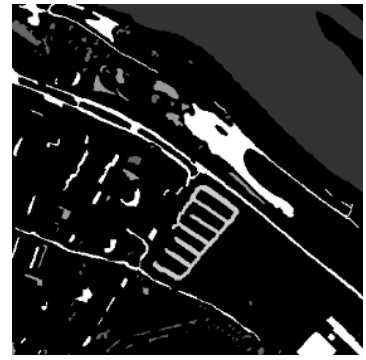

b)

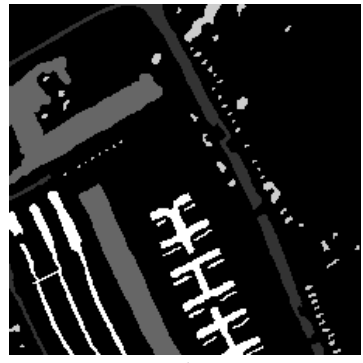

c)

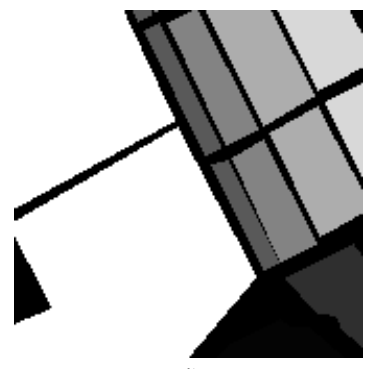

d)

Figure 3. Examples of the reference classification masks for images: a) Botswana-1, b) Pavia-1, c) PaviaU-1,

d) Salinas-1. Black color is used for data without class label

\section{EXPERIMENTAL RESULTS}

\subsection{Estimation of Fusion Influence on Classification Quality}

To estimate how the fusion affects classification quality, we classified the fused image and average interpolated input LR images $Z_{k}$ for each imaging system. The classification results for SVM and RF algorithms are shown in tables 5 and 6 correspondingly.

The data in table 5 show that for the SVM algorithm, the classification accuracy of the fused image was slightly worse than the classification accuracy of the average images for each system. The fusion results were classified with the average accuracy of 76.16, whereas for the system 1 and system 2, the classification accuracy was equal to 78 and 76.56 correspondingly. Thus, the smoother representation obtained after interpolation and averaging is better for SVM than a more detailed representation of the fused image.

Table 6 demonstrates that for the RF classification, the average classification accuracy of the fused image is higher than the accuracy of the images for each imaging system in $90 \%$ of the cases. Since system 1 had the smallest difference in spatial resolution with the fusion results, the results of system 1 image classification are the closest to the fused image classification results. Nevertheless, the classification accuracy of the fusion result was $4 \%$ higher than the classification accuracy of the system 1 image. Thus, the super-resolution fusion enhances the performance of the RF classifier.

Table 5. Accuracy of SVM classification

\begin{tabular}{lrrrrrr}
\hline Image & Fusion result & \multicolumn{1}{c}{ system 1 } & \multicolumn{1}{c}{ system 2 } & \multicolumn{1}{c}{ system 3 } & \multicolumn{1}{c}{ system 4 } & Reference image \\
\hline Botswana-1 & 89.84 & $\mathbf{8 9 . 8 7}$ & 77.11 & 78.98 & 79.33 & 87.18 \\
Botswana-2 & 75.51 & 73.25 & $\mathbf{8 2 . 7 5}$ & 75.71 & 73.22 & 87.68 \\
Pavia-1 & 71.74 & 76.43 & $\mathbf{7 6 . 9 3}$ & 69.84 & 60.90 & 82.44 \\
Pavia-2 & 63.44 & 70.08 & $\mathbf{7 6 . 6 2}$ & 70.05 & 65.68 & 77.30 \\
Pavia-3 & 77.44 & 85.19 & $\mathbf{8 5 . 3 3}$ & 77.93 & 53.10 & 78.54 \\
PaviaU-1 & 79.71 & 78.83 & $\mathbf{8 1 . 5 8}$ & 75.48 & 63.54 & 74.88 \\
PaviaU-2 & 63.61 & 66.67 & 57.02 & $\mathbf{6 7 . 5 8}$ & 62.82 & 69.00 \\
PaviaU-3 & $\mathbf{7 0 . 2 5}$ & 66.55 & 59.24 & 65.16 & 65.79 & 70.59 \\
Salinas-1 & 80.16 & $\mathbf{8 3 . 2 6}$ & 75.16 & 81.34 & 78.56 & 83.14 \\
Salinas-2 & 89.92 & 89.84 & $\mathbf{9 3 . 8 3}$ & 92.83 & 91.75 & 90.90 \\
\hline Total average & $\mathbf{7 6 . 1 6}$ & $\mathbf{7 8 . 0 0}$ & $\mathbf{7 6 . 5 6}$ & $\mathbf{7 5 . 4 9}$ & $\mathbf{6 9 . 4 7}$ & $\mathbf{8 0 . 1 7}$ \\
\hline
\end{tabular}


Table 6. Accuracy of RF classification

\begin{tabular}{lrrrrrr}
\hline Image & Fusion result & \multicolumn{1}{c}{ system 1 } & system 2 & system 3 & system 4 & Reference image \\
\hline Botswana-1 & $\mathbf{8 5 . 8 5}$ & 79.08 & 71.46 & 74.40 & 68.45 & 83.97 \\
Botswana-2 & $\mathbf{8 6 . 4 3}$ & 81.64 & 86.44 & 82.74 & 86.17 & 81.82 \\
Pavia-1 & $\mathbf{8 1 . 3 5}$ & 79.02 & 77.95 & 80.76 & 78.85 & 79.47 \\
Pavia-2 & $\mathbf{8 2 . 2 7}$ & 74.72 & 79.53 & 77.16 & 74.35 & 84.17 \\
Pavia-3 & $\mathbf{8 6 . 3 7}$ & 83.35 & 78.63 & 81.63 & 75.13 & 85.08 \\
PaviaU-1 & 81.84 & $\mathbf{8 5 . 0 1}$ & 78.91 & 79.39 & 74.87 & 90.42 \\
PaviaU-2 & $\mathbf{6 4 . 2 5}$ & 61.95 & 48.46 & 56.17 & 53.24 & 64.09 \\
PaviaU-3 & $\mathbf{7 2 . 8 0}$ & 68.94 & 54.04 & 60.87 & 59.02 & 70.72 \\
Salinas-1 & $\mathbf{8 3 . 4 1}$ & 81.58 & 80.13 & 77.24 & 77.92 & 85.17 \\
Salinas-2 & $\mathbf{8 8 . 4 1}$ & 87.63 & 83.54 & 85.48 & 85.09 & 88.89 \\
\hline Total average & $\mathbf{8 1 . 3 0}$ & $\mathbf{7 8 . 2 9}$ & $\mathbf{7 3 . 9 1}$ & $\mathbf{7 5 . 5 8}$ & $\mathbf{7 3 . 3 1}$ & $\mathbf{8 1 . 3 8}$ \\
\hline
\end{tabular}

Overall, the results of the fusion with RF classification were better than the ones for SVM classifier. The average classification accuracy of the fused image with RF classifier was 81.3 in comparison with the best average classification accuracy of the SVM method equal to 78.

\subsection{The Influence of Feature Extraction Methods on the Fused Image Classification}

The experimental research of the feature extraction methods was performed only for RF classification of the fused image. The following feature extraction methods were considered:

1) Brightness in spectral channels,

2) Extended Attribute Profiles (EAP) (Pham et al., 2018),

3) Local Feature Attribute profiles (LFAP) (Pham et al., 2017).

EAP is constructed using different attribute filters applied to each spectral channel of the image. Attribute filters are the generalization of morphological filters with the appropriate criterion function of the connected components of the image. The threshold values of the criterion define a set of attribute filters. For example, the area attribute filter with the predefined threshold removes all connected components of the image having the area smaller than the threshold.

LFAP is formed from EAP by means of additional local filtration of EAP features. According to paper (Pham et al., 2017), both methods are very effective for hyperspectral remote sensing images. In (Pham et al., 2017) it was also considered the case of dimension reduction methods because hyperspectral images have hundreds of spectral channels. In our case, the fusion result is a multispectral image and there is no need for dimension reduction. We calculated EAP and LFAP for spectral brightness values of the fused image. We used EAP features proposed in (Pham et al., 2017) for the classification of Pavia University image (Graña et al., 2020). The EAP feature description is given in Table 7.

Table 7. The parameters of the EAP

\begin{tabular}{ll}
\hline Attribute & Threshold \\
\hline Square & $100,500,1000,5000$ \\
Standard deviation & $20,30,40,50$ \\
Moment of inertia & $0.2,0.3,0.4,0.5$ \\
Diagonal & $10,25,50,100$ \\
\hline
\end{tabular}

As for LFAP features, we computed them from the EAP using local average and local range filers with the window size equal to $7 \times 7$.

The RF classification results using different feature extraction methods are shown in Table 8 . 
Table 8. RF classification accuracy using different feature extraction methods

\begin{tabular}{|c|c|c|c|}
\hline Image & EAP & brightness & LFAP \\
\hline Botswana-1 & 82.04 & 83.55 & 78.07 \\
\hline Botswana-2 & 95.11 & 86.49 & 85.48 \\
\hline Pavia-1 & 84.11 & 80.33 & 80.21 \\
\hline Pavia-2 & 90.03 & 84.31 & 77.24 \\
\hline Pavia-3 & 96.46 & 77.23 & 81.43 \\
\hline PaviaU-1 & 91.51 & 86.63 & 80.94 \\
\hline PaviaU-2 & 68.50 & 63.11 & 60.53 \\
\hline PaviaU-3 & 79.88 & 68.01 & 74.76 \\
\hline Salinas-1 & 92.84 & 83.23 & 82.25 \\
\hline Salinas-2 & 94.41 & 90.94 & 88.26 \\
\hline Total average & 87.49 & 80.38 & 78.92 \\
\hline
\end{tabular}

The best choice for the RF algorithm is EAP features. The classification accuracy of the fused image using EAP is from 70 to $95 \%$ and it is higher on $9 \%$ than in the case without EAP. LFAP does not increase classification accuracy in comparison to the brightness features.

Thus, it can be concluded that RF classification of the fused image is more effective if the EAP features are used.

The examples of the RF classification with fusion and without fusion are shown in figures 4-7 correspondingly.

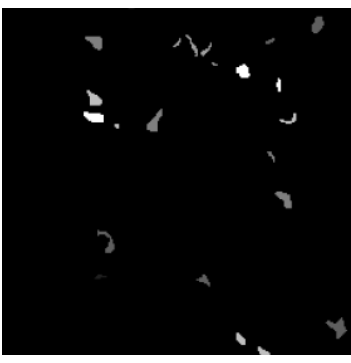

a)

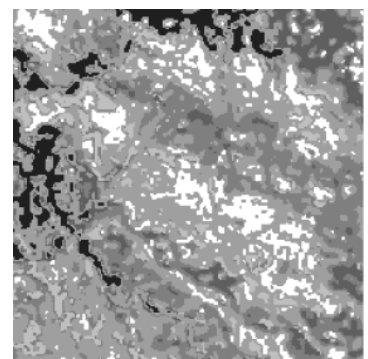

b)

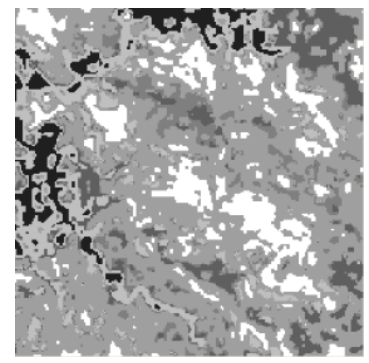

c)

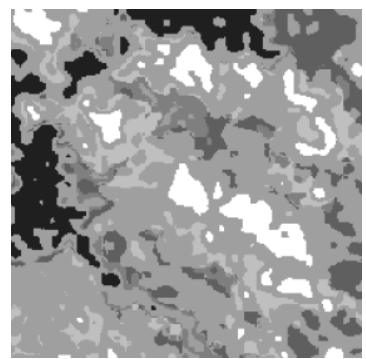

d)

Figure 4. Reference classification mask (a) and examples of the RF classification results for Botswana-1: b) fused image classification, c) system 1 classification, d) system 3 classification

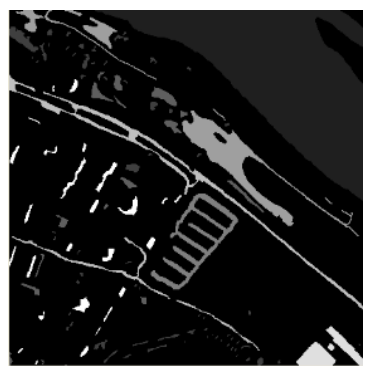

a)

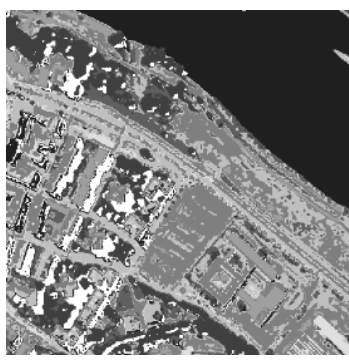

b)

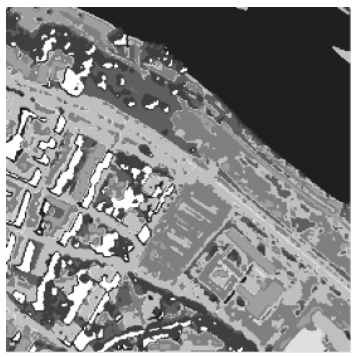

c)

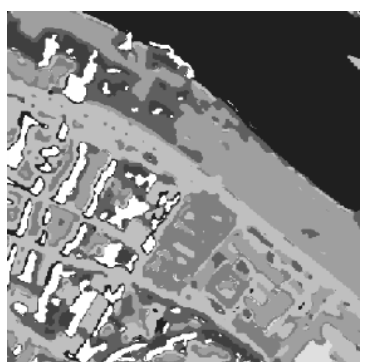

d)

Figure 5. Reference classification mask (a) and examples of the RF classification results for Pavia-1: b) fused image classification, c) system 1 classification, d) system 3 classification 


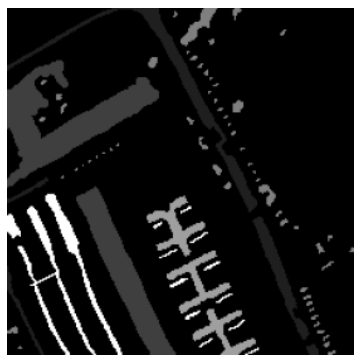

a)

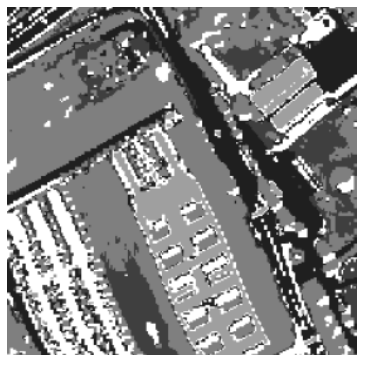

b)

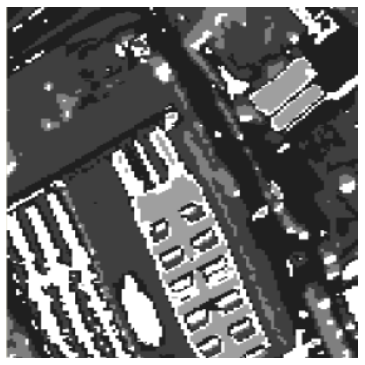

c)

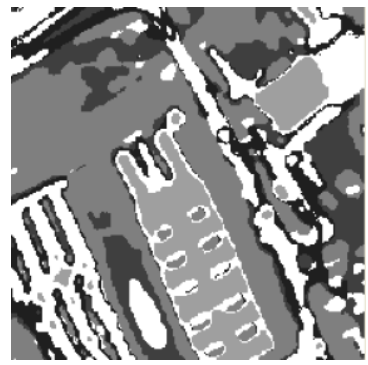

d)

Figure 6. Reference classification mask (a) and examples of the RF classification results for PaviaU-1: b) fused image classification, c) system 1 classification, d) system 3 classification

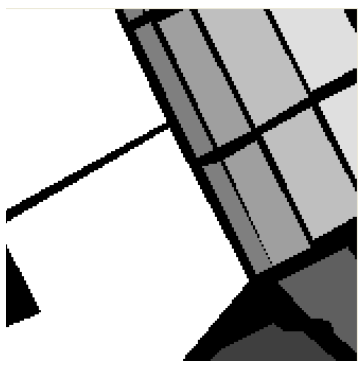

a)

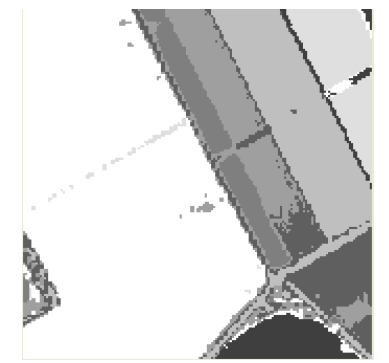

b)

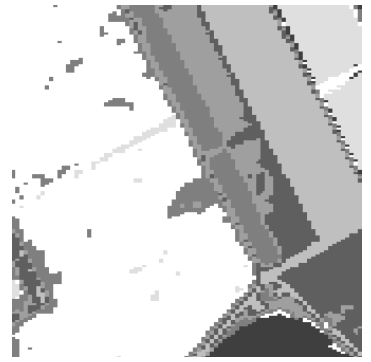

c)

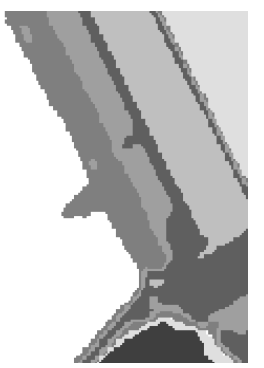

d)

Figure 7. Reference classification mask (a) and examples of the RF classification results for Salinas-1: b) fused image classification, c) system 1 classification, d) system 3 classification

Thus, super-resolution image fusion enhances the results of RF classification whereas SVM classification is better to use without fusion. The reason for this is that SVM performs better on the smother images whereas RF needs extra spatial and spectral details for better performance.

\section{CONCLUSION}

The paper concerns the question of the spectral-spatial super-resolution image fusion influence on the classification of remote sensing images. The experiments were conducted for simulated remote sensing images of four imaging systems with GSDs in 2, 3, 4 and 5 times larger than the GSD of the fused image. The fused image included 11 or 15 spectral channels with the average bandwidth of $29 \mathrm{~nm}$ according to the spectral ranges of the input images. Spectral channels of the simulated low resolution images included from 3 to 8 spectral channels with average bandwidth from $37 \mathrm{~nm}$ to $83 \mathrm{~nm}$. Our experimental research showed that SVM classification is better to apply without fusion because the SVM performance for the smooth interpolated images is better than for the fusion result which has more detailed image representation. In contrast to SVM, RF classification of the fused image produces in $90 \%$ of the cases better results than without fusion. For example, for the image with spatial resolution two times worse than the spatial resolution of the fused image, the classification accuracy of the fused image was $4 \%$ higher. The classification results obtained for RF classifier with fusion are better than the classification results for SVM without fusion. In particularly, the average classification accuracy for the fusion with RF classification was 81.3 whereas the best classification accuracy of SVM without fusion was 78. The experiment with different feature extraction methods showed that extended attribute profiles are preferable for RF classification with fusion. The accuracy of the fused image classification with RF algorithm and EAP were on $9 \%$ higher than in the case of brightness features.

Thus, the best classification is obtained for the RF classifier with fusion whereas there is no need to apply fusion with SVM algorithm. 


\section{ACKNOWLEDGEMENT}

The research was supported by RFBR project №18-07-00748.

\section{REFERENCES}

Belgiu, M. and Drăguț, L., 2016. "Random forest in remote sensing: A review of applications and future directions" ISPRS Journal of Photogrammetry and Remote Sensing, vol. 114, pp. 24-31.

Belov, A.M. and Denisova, A.Y., 2018. "Spectral and spatial super-resolution method for Earth remote sensing image fusion" Computer Optics, vol. 42, pp. 855-863.

Farsiu, S., Robinson, M.D., Elad, M. and Milanfar, P., 2003. "Fast and robust super-resolution" Proceedings of the 2003 International Conference on Image Processing, vol. 3, pp. 291-294.

Farsiu, S., Robinson, M.D., Elad, M. and Milanfar, P., 2004. "Fast and robust multiframe super resolution" IEEE Transactions on Image Processing, vol. 13, pp. 1327-1344.

García, M.A., Moutahir, H., Casady, G.M., Bautista, S. and Rodríguez, F., 2019. "Using Hidden Markov Models for Land Surface Phenology: An Evaluation Across a Range of Land Cover Types in Southeast Spain" Remote Sensing, vol.11, No.5.

Graña, M., Veganzons, M.A. and Ayerdi, B. 2020. "Hyperspectral Remote Sensing Scenes", available at http://www.ehu.eus/ccwintco/index.php?title=Hyperspectral_Remote_Sensing_Scenes

Hong, D., Wu, X., Ghamisi, P., Chanussot, J., Yokoya, N. and Zhu, X.X., 2020. "Invariant attribute profiles: A spatialfrequency joint feature extractor for hyperspectral image classification" IEEE Transactions on Geoscience and Remote Sensing, pp. 1-18.

Khatami, R., Mountrakis, G. and Stehman, S.V., 2016. "A meta-analysis of remote sensing research on supervised pixel-based land-cover image classification processes: General guidelines for practitioners and future research" Remote Sensing of Environment, vol.177, pp. 89-100.

Li, J., Huang, X., Gamba, P., Bioucas-Dias, J.M., Zhang, L., Benediktsson, J.A. and Plaza, A. 2015. "Multiple Feature Learning for Hyperspectral Image Classification" IEEE Transactions on Geoscience and Remote Sensing, vol. 53, pp. 1592-1606.

Li, M., Ma, L., Blaschke, T., Cheng, L. and Tiede, D., 2016. "A systematic comparison of different object-based classification techniques using high spatial resolution imagery in agricultural environments" International Journal of Applied Earth Observation and Geoinformation, vol. 49, pp. 87-98.

Liao, W., Dalla Mura, M., Chanussot, J. and Pižurica, A., 2015. "Fusion of spectral and spatial information for classification of hyperspectral remote-sensed imagery by local graph" IEEE Journal of Selected Topics in Applied Earth Observations and Remote Sensing, vol. 9, pp. 583-594.

Marpu, P.R., Pedergnana, M., Dalla Mura, M., Benediktsson, J.A. and Bruzzone, L., 2013. "Automatic Generation of Standard Deviation Attribute Profiles for Spectral-Spatial Classification of Remote Sensing Data" Geoscience and Remote Sensing Letters, vol. 10, pp. 293-297.

Pham, M.T., Aptoula, E., and Lefèvre, S., 2018. "Classification of remote sensing images using attribute profiles and feature profiles from different trees: a comparative study" IGARSS 2018-2018 IEEE International Geoscience and Remote Sensing Symposium, pp. 4511-4514.

Pham, M.T., Lefèvre, S., and Aptoula, E., 2017. "Local feature-based attribute profiles for optical remote sensing image classification" IEEE Transactions on Geoscience and Remote Sensing, vol. 56, pp. 1199-1212.

Pham, M.T., Lefèvre, S., Aptoula, E. and Bruzzone, L., 2018. "Recent developments from attribute profiles for remote sensing image classification" arXiv preprint arXiv:1803.10036 2018.

Tuia, D., Volpi, M., Dalla Mura, M., Rakotomamonjy, A. and Flamary, R., 2014. "Automatic feature learning for spatio-spectral image classification with sparse SVM" IEEE Transactions on Geoscience and Remote Sensing, vol. 52, pp. 6062-6074. 